\title{
La e-langue des jeunes en France et en Ukraine
}

\author{
The e-language of the young in France and in Ukraine
}

\author{
Andriy Bilas \\ Université Nationale Prycarpatsky Vasyl Stefanyk, Ukraine
}

\begin{abstract}
Résumé : Le cadre de cet article est l'étude comparative des nouvelles formes de la langue des jeunes induites par le développement de la communication par Internet et son impact sur la communication et la langue communes. Notre étude s'appuie également sur toute une littérature qui cherche à penser les formes de la langue des jeunes à partir de l'analyse des marqueurs de la e-langue des jeunes en France et en Ukraine afin de dégager la cohérence sociolinguistique des universaux de ce sociolecte. Une approche pluridisciplinaire permet de construire une typologie des formes de la e-langue des jeunes.
\end{abstract}

Mots-clés : e-langue des jeunes, sociolecte, formes, marqueurs, typologie, universaux.

Abstract: The article proposes the comparative study of the new forms of youth's slang induced by the development of communication via Internet and its impact on common communication and language. Our study also considers scientific literature examining e-language markers of French and Ukrainian youth striving for sociolinguistic cooperation under the analyzed sociolect circumstances. The multidisciplinary approach makes it possible to construct the typology of the youth's e-language forms.

Keywords: youth e-language, sociolect, forms, markers, typology, universals.

\section{Introduction}

Ce travail propose une approche typologique des ressources lexicales de la elangue des jeunes dans deux espaces linguistiques différents. Nous poursuivons l'objectif de comparer différents marqueurs de la e-langue des jeunes et, derrière cette typologie, de comprendre la perception que peuvent en avoir de jeunes internautes.

Ancrée dans le contexte de la sociolinguistique, la présente recherche vise à comprendre comment la communication par Internet provoque l'évolution de la langue commune des jeunes en France et en Ukraine. La confrontation des usages des jeunes de ces deux pays permet d'identifier les nouvelles formes liées à une émotion ou à un sentiment.

Depuis plusieurs années, les nouvelles formes de production linguistique liées à la communication par Internet ont intéressées de nombreux chercheurs (Anis, 2003 ; Barthélémy, 2007 ; Chemerkin, 2009 ; Dejond, 2002 ; Baldino Putzka, Boutin, 2012 ; 
Fiévet, 2010 ; Gajos, 2006 ; Kondratyuk, 2005 ; Ostapenko, 2013 ; Podhorná-Polická, 2008 ; Yaroshchuk, 2012).

\section{La langue non-standard des jeunes}

La e-langue des jeunes, en principe, entre dans le corpus de la langue non-standard des jeunes. La langue non-standard des jeunes est un phénomène lexical qu'on ne traite pas comme une substance linguistique homogène, et qui comprend l'argot commun des jeunes et leurs argots spéciaux des jeunes. En général, l'argot des jeunes correspond à la langue d'une certaine génération, laquelle est dynamique par nature et soumise, avec son système lexical, au changement constant de la mode verbale.

Les argots des jeunes (argot commun des jeunes, ainsi que l'argot commun des jeunes des cités) ne sont pas entièrement répertoriés dans les dictionnaires d'usage, alors qu'ils sont couramment utilisés par les jeunes en général, les jeunes qui s'autoidentifient avec la «culture des rues ». Selon A.-C. Fiévet :

Ce lexique est souvent inconnu des dictionnaires, même des dictionnaires spécialisés dans l'argot des jeunes et on a affaire à une sorte de néologie mi-idiolectale, mi-sociolectale (Fiévet, 2010 : 986).

En comparant les travaux sur la production verbale des jeunes en linguistique étrangère, on constate que certains chercheurs sont orientés traditionnellement vers la sociologie, tandis que d'autres appuient davantage leurs théories sur une approche psychologique. D'autres encore (Fiévet, 2008 : 23) s'adonnent au travail comparatif qui consiste en l'application des deux approches citées précédemment afin d'en tirer des conclusions communes pour tous les jeunes.

Chaque nouvelle génération se distingue, consciemment ou inconsciemment, de la précédente par appréciation différente des valeurs de la vie :

Les nouvelles vagues sont souvent considérées comme provocantes ou, tout du moins, choquantes par leur non-conformisme, mais elles fascinent en même temps par leur charge créatrice - novatrice. [...] Le maniement des ordinateurs, des portables, etc. est une évidence pour les jeunes ainsi que l'usage des néologismes créés pour décrire cette nouvelle réalité (Podhorná-Polická, 2008 : 24).

De là le fait que la langue des jeunes, surtout la e-langue, devienne un outil qui reflète bien ce paradoxe :

[...] le potentiel créatif néologique d'une part, la fascination pour l'argot (au sens classique du mot référent à des milieux associaux) et pour d'autres formes transgressives, nonconventionnelles que la langue propose d'autre part, sont les deux traits les plus saillants si l'on se pose la question des particularités propres à l'expression verbale des jeunes (Podhorná-Polická, 2008 : 24).

On définit la langue des jeunes comme un sociolecte. Pourtant, sa définition a évolué de manière significative avec l'essor des nouvelles technologies, ce qui a pour conséquence un changement de paradigme en linguistique. Les communautés d'échange en ligne ont recours à des codes sociolectaux. L. Goudet appelle " communaulectes " ces sociolectes apparus sur Internet. Ils s'appuient sur 
un rayonnement mémétique. La dimension linguistique des mèmes de l'écrit est tout à fait novatrice : elle est due au média lui-même (Goudet, 2016).

On est sûr que les jeunes sont les plus concernés par la communication par Internet, puisqu'ils sont en quête de leur identité, encore immature et fragile face à chaque " inintelligence » et maladresse dans la présentation de leur univers culturel (Podhorná-Polická, 2007 : 12).

On s'accorde avec A. Podhorná-Polická pour dire que la langue des jeunes est un thème qui se prête facilement à la commercialisation, puisqu'il attire non seulement les jeunes eux-mêmes mais aussi les adultes en quête de débouchés pédagogiques, y compris des chercheurs en sciences humaines et sociales pour lesquels la médiatisation facilite l'accès à des réseaux par ailleurs difficiles à pénétrer.

La langue des jeunes se caractérise par son caractère oral, mais ce qui nous intéresse particulièrement, c'est son caractère écrit tel qu'il se présente dans la correspondance privée, sous forme de mails ou de textos, ou semi-privée, sous forme de chats, commentaires, forum, etc., avec une diversité territoriale importante. Le critère diatopique est étudié dans le milieu étudiant ukrainien où des variantes, notamment dans l'écriture oralisée, permettent de différencier la base dialectale, sociolectale des jeunes. Dans les productions écrites spontanées des étudiants de la faculté des langues étrangères à l'Université nationale Prycarpatsky (Ivano-Frankivsk, Ukraine), issus de différentes régions de l'Ukraine, nous observons par exemple une insertion de la flexion appartenant à un parler des jeunes, exagérée dans les échanges ironiques ou ludiques.

En revanche, en France, les tendances à donner des particularités diatopiques à la langue des jeunes se limitent à la différence lexicale. D'une part, la forte cohésion des groupes de jeunes empêche la transmission des néologismes et contribue à une très forte variabilité lexicale, et d'autre part, les médias imposent l'échange verbal de tous les jeunes et aident à les unifier. De plus, les médias contribuent à la stéréotypisation des jeunes qui acceptent volontairement de se différencier des autres, y compris par les modes langagiers (Podhorná-Polická, 2007 : 110).

\section{La e-langue comme forme de communication des jeunes}

L'émergence de la e-langue des jeunes est étudiée à partir de travaux portant sur la dynamique d'évolution d'un langage, analysée sous l'angle de sa fonction communicationnelle (aspects stratégiques, sociaux), d'une part, et d'autre part, sous l'angle de sa fonction informative (efficacité de la transmission, utilité).

En même temps, la e-langue comme forme de communication des jeunes relativement récente se caractérise paradoxalement par des contraintes, dues à la spécification technique et des interfaces de saisie, et par une liberté remarquable qu'elle tire d'un registre de communication familier, supportant les codes d'un groupe restreint (Barthélémy 2007). J. Anis décrit ainsi le développement d'un langage réseau, d'une nouvelle variété du français écrit. II s'agit d'un écrit :

- brut (sans relecture) ;

- familier (alors qu'on associe habituellement écrit et formalisme) ;

- affectif (expression des sentiments favorisant le relâchement du contrôle) ; 
- Iudique (s'exprimant par la néographie, le jeu de mot) ;

- socialisant (dominance de la fonction phatique dans les messages, partage de codes communs) (Anis, 2003).

Communiquer en français sur Internet demande une initiation solide en cyberlangue, or celle-ci, pour A. Déjond:

[...] n'est pas une sous-langue, mais un langage parallèle [...], une sorte de mélange entre l'oral et l'écrit, un style oratoire bousculé par la vitesse, chamboulé dans ses règles et ses conventions (Dejond, 2002 : 18).

Selon M. Baldino Putzka et E. Boutin, la cyberlangue correspond à une nouvelle forme de langage, efficace, rapide, inventif, ludique et partagé par une communauté d'utilisateurs (Baldino Putzka, Boutin, 2012 : 2). La rapidité des échanges verbaux sur Internet et le nombre limité de signes linguistiques à utiliser causent toutes sortes de modifications et de créations nouvelles au niveau lexical ainsi qu'au niveau morphosyntaxique. Voilà pourquoi M. Gajos met en avant la base parlée du cyberlexique :

\begin{abstract}
Certaines formes du lexique sont empruntées directement au français parlé courant et populaire, d'autres ont été créées spécialement pour satisfaire les besoins de communication dans le cyberespace. On peut facilement trouver dans les énoncés des natifs échangés sur le net des unités lexicales et des structures d'énoncés spécifiques au cyberlangage français (Gajos, 2006 : 173).
\end{abstract}

Pourtant, M. Gajos n'a pas été le premier à faire cette observation. Déjà, au tout début des recherches dans le domaine de la cyber-linguistique, J. Anis avait remarqué qu'en fait, les effets d'oralité sont à la base des caractéristiques formelles de ce parlécrit, comme l'abréviation, l'iconicité, notamment à travers les émoticônes. Mais ces caractéristiques peuvent entrer en contradiction: les étirements graphiques, phénomène d'iconicité, offrent ainsi un contraste évident avec les abréviations (Anis, 2003).

\title{
3. Marqueurs de la e-langue des jeunes
}

Dans notre article, on se base sur la typologie des marqueurs de la e-langue des jeunes proposée par J. Anis. Le linguiste différencie deux aspects des marqueurs : la variation graphique, et quelques aspects morpho-lexicaux. Selon J. Anis, la variation graphique comprend les néographies, c'est-ò-dire, toutes les graphies s'écartant de la norme orthographique, nommées aussi graphies phonétisantes (réductions graphiques, réductions avec variantes phonétiques, squelettes consonantiques, syllabogrammes, rébus à transfert, logogrammes, paralogogrammes, étirements graphiques et combinaisons de plusieurs procédés) et les particularités morpholexicales comme la troncation, l'emploi des anglicismes adoptés, le verlan et les onomatopées (Anis, 2003).

On utilise un corpus de messages fournis par des étudiants français et ukrainiens et qui ont été recueillis sur Facebook pour cette recherche. On comparera la présence de tel ou tel phénomène dans ces messages pour saisir la spécificité des modes de communication des utilisateurs de chacune de ces langues, et des visions du monde correspondantes. 
On est sûr que le système d'écriture orthographique se traduit par la présence de signes muets en fin de mot. Dans la e-langue, ces signes muets sont supprimés, ce qui fait de cet idiome une écriture entre l'écrit et l'oral. J. Anis parle de "parlécrit» (Anis, 1999 : 74), d'une forme d'écriture qui remet en cause la distinction entre l'oral et le scriptural et rend indispensable une redéfinition du rapport discours oral/discours écrit (Baldino Putzka, Boutin, 2012 : 2).

A. Dejond remarque bien qu'on ne peut pas définir absolument les règles de la formation des unités langagières, car elles changent, elles sont dynamiques :

Le cyberlangage est plutôt une variété la langue: des principes (abréger, tronquer, sigler, ajouter des émoticônes, s'exprimer par onomatopées), mais pas de règles (on ne peut pas parler d'une véritable unification du cyberlangage, puisque selon l'internaute, les déclinaisons d'écriture changent. Par exemple, " je sais " peut être traduit par " je sé ", " je C ", " ché », " chais » etc. (Dejond 2006:11).

Dans le corpus de l'écriture électronique, M. Baldino Putzka et E. Boutin identifient comme procédés-marqueurs de la cyberlangue des figures de style principalement issues de la linguistique, et des procédés d'écriture issus des sciences de l'éducation, en précisant l'utilisation de multiples combinatoires intégrant, le plus souvent, deux à trois procédés d'écriture différents, et en mettant en évidence le fort potentiel des marqueurs de la cyberlangue. Les chercheurs catégorisent ainsi six dynamiques communicationnelles, respectivement sous-catégorisées en procédés d'écriture purs ou en combinatoires: alternatives graphiques (étirement graphique, agglutination, euphonie, graphie phonétisante, réduction graphique, graphie serpentin, graphie isochrone, onomatopées), suppressions de phonèmes (troncations, sigles, rébus, acronymes, syllabogrammes, logogrammes, squelettes consonantiques, chute de mutogrammes), emprunts à d'autres langues (anglicisme, italianisme, hispanisme, hellénisme), termes conceptuels interactifs (verlan, émoticônes, antonomases, motvalise), variations de phonèmes isolés ou multiples (alternance vocalique, alternance consonantique, substitution de consonne, écrasement phonétique) et ajouts de phonèmes (gémination, paragoge, épenthèse, prosthèse) (Baldino Putzka, Boutin, $2012: 3)$.

\section{Emploi motivé de la e-langue des jeunes}

Les marqueurs de la e-langue correspondent à différentes motivations chez les jeunes qui les emploient : lisibilité, niveau de langue, expression de la pensée et des sentiments, utilisation de la métaphore (Baldino Putzka, Boutin, 2012 : 2).

L'analyse des sources théoriques et les résultats d'une enquête effectuée auprès des étudiants de la faculté des langues étrangères à l'Université nationale Prycarpatsky (Ivano-Frankivsk, Ukraine) ont permis d'établir les principales raisons de l'utilisation de la e-langue des jeunes:

Premièrement, les étudiants utilisent les cybermots pour avoir l'air moderne. Lors de la communication, ils utilisent le cyberlexique non seulement pour transmettre des informations, mais aussi pour exprimer leur propre vision du monde, leurs idées, pour mettre en évidence leur personnalité de façon dynamique.

Deuxièmement, la e-langue est une forme de "perfusion " au sein d'un groupe, un moyen de sélectionner la perception adéquate: en effet, les étudiants font 
seulement usage de la e-langue dans des situations où ils veulent qu'on les comprenne. Presque toujours, les personnes interrogées nient le fait qu'elles utilisent délibérément le cyberlexique, affirmant que cela se produit inconsciemment.

Troisièmement, les jeunes utilisent la e-langue dans un contexte particulier. On peut entendre la plupart de ces expressions électroniques au cours d'une cyberconversation dans un cercle d'amis, entre les camarades d'un même groupe, parce que ces expressions servent à les rapprocher émotionnellement les uns des autres et les aident à exprimer facilement, et de manière concise, leurs attitudes face aux événements ou aux phénomènes discutés.

Quatrièmement, la e-langue peut être utilisée par une sorte de jeu pour décrire de façon précise, même ironique des événements et des impressions. Elle permet de donner à la communication un caractère d'intimité et recouvre alors une fonction sociale importante, favorisant l'entrée dans un groupe donné.

À notre avis, un groupe de jeunes emploie invariablement des lexèmes dans sa ecommunication spontanée, en France aussi bien qu'en Ukraine, qu'ils soient classés sous une marque argotique, vulgaire, populaire, ou familière. Ce qui importe surtout, c'est leur acception identitaire, ludique et conniventielle par les membres du groupe, et leur expressivité (Anis, 2003 ; Boyer, 2001 ; Baldino Putzka, Boutin, 2012 ; Sourdot, 2003 ; Beregovskaya, 1996).

Les jeunes de tous les milieux tendent à nommer les choses ou les faits de façon innovatrice, non-conformiste, choquante, essayant de rafraîchir des thèmes répétitifs par des lexèmes nouveaux. C'est là une des caractéristiques stables de la langue des jeunes, toutes générations confondues, même si son contenu (néologie) est d'une labilité maximale (Podhorná-Polická, 2006: 99). F. Gadet remarque à ce propos que :

[...] la stratification en âge peut indiquer un changement en cours, mais peut aussi montrer une évolution en cours de vie, qui se répéterait génération après génération (Gadet, 2003 : 68).

\section{Analyse comparative des corpus français et ukrainien de la e-langue}

Le cadre général de ce travail a été l'étude des nouvelles formes de langue induites par le développement des technologies de l'information et de la communication. La e-langue est un genre textuel qui s'émancipe de l'écriture conventionnelle. Cette nouvelle forme langagière est axée sur la combinatoire de plusieurs procédés d'écriture, faisant appel aux aspects graphiques du traitement de la langue écrite.

Dans ce travail, nous poursuivons un double objectif. II s'agit d'isoler et de comparer différents marqueurs des e-langues française et ukrainienne.

Nous nous focalisons sur les messages des jeunes sur Facebook et Viber en $2016-$ 2018 en nous attachant surtout aux formes linguistiques. En français, l'orthographe et la prononciation des unités langagières ne coïncident pas, alors qu'en ukrainien les mots comprennent le même nombre de lettres et de sons. Donc, si les jeunes internautes français ont tendance à écrire comme ils parlent, en transcrivant avec les lettres de l'alphabet latin " leur prononciation », les jeunes internautes ukrainiens n'ont pas besoin de le faire. Voilà pourquoi le discours des jeunes Ukrainiens possède peu de procédés de graphies phonétisantes pour former les mots nouveaux en 
comparaison de celui des jeunes internautes français (exemples dans le tableau cidessous).

Tableau comparatif du corpus français et ukrainien :

\begin{tabular}{|c|c|c|}
\hline Procédés & Exemples français & Exemples ukrainiens \\
\hline $\begin{array}{l}\text { réductions } \\
\text { graphiques }\end{array}$ & $q u=k$ & - \\
\hline $\begin{array}{l}\text { substitutions } \\
\text { graphiques }\end{array}$ & kler = clair & - \\
\hline chute des e instables & douch froid, fet = fête & - \\
\hline $\begin{array}{l}\text { chute des } \\
\text { mutogrammes en } \\
\text { finale }\end{array}$ & cour(s) & - \\
\hline $\begin{array}{l}\text { simplification des } \\
\text { digrammes et } \\
\text { trigrammes }\end{array}$ & ossi $=$ aussi, bo $=$ beau & - \\
\hline $\begin{array}{l}\text { déconstruction de } \\
\text { «oi» }\end{array}$ & mova $=$ moi & - \\
\hline $\begin{array}{l}\text { réductions avec } \\
\text { compactage }\end{array}$ & $\begin{array}{l}\text { cé = c'est, keske = qu'est-ce } \\
\text { que, keski = qu'est-ce qui, } \\
\text { mapelé = m'appeler, jsui = je } \\
\text { suis, kekina = qu'est ce qui y } \\
\text { a }\end{array}$ & - \\
\hline $\begin{array}{l}\text { remplacement des } \\
\text { graphèmes par le -é }\end{array}$ & $\begin{array}{l}\text { fé }=\text { fait, cé/sé = } \\
\text { c'est/sais/sait, mé = mais, vé } \\
=\text { vais }\end{array}$ & - \\
\hline $\begin{array}{l}\text { acronymes, sigles et } \\
\text { abréviations }\end{array}$ & $\begin{array}{l}\text { PTI = pour ton information), } \\
\text { ALP = à la prochaine), taf = } \\
\text { travail à faire, AMA = à mon } \\
\text { avis, AMHA = à mon humble } \\
\text { avis, ASV = âge, sexe, ville, } \\
\text { BAP = bon après-midi, CAD = } \\
\text { C'est-à-dire, Cki ? = on se } \\
\text { connaît, CPG = c'est pas } \\
\text { grave, DDD = demande de } \\
\text { discussion, DQP = dès que } \\
\text { possible, ENTK = en tout cas, } \\
\text { EXPDR = explosé de rire, FDS = } \\
\text { fin de semaine, }\end{array}$ & 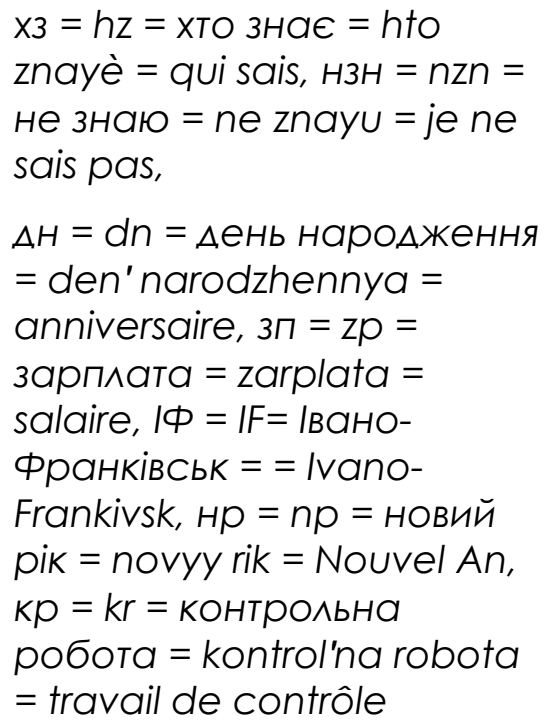 \\
\hline
\end{tabular}




\begin{tabular}{|c|c|c|}
\hline $\begin{array}{l}\text { écrasements } \\
\text { phonétiques }\end{array}$ & $\begin{array}{l}\text { chais = je sais } \\
\text { ayé = ça y est, } \\
\text { chui = je suis, }\end{array}$ & $\begin{array}{l}\text { MOHa = mona = можна = } \\
\text { mozhna = on peut, шо = sho } \\
=\text { що = shcho = quoi, шось } \\
=\text { shos' = щось = shchos' = } \\
\text { quelque chose, we = she = } \\
\text { щe = shche = encore, kapo4 } \\
=\text { karoch = kоpoчe = } \\
\text { koroche = bref, чo = cho = } \\
\text { що = shcho = quoi }\end{array}$ \\
\hline $\begin{array}{l}\text { valeur phonétique } \\
\text { des lettres }\end{array}$ & $\begin{array}{l}\text { je } V \text { manG = je vais manger, } \\
\text { NRV = énervé, jtm = je t'aime, } \\
\text { aPRO = apéro, } A \Pi=\text { à tout à } \\
\text { l'heure, C Ca = c'est ça, KDO } \\
=\text { cadeau, } G=j \text { 'ai, } T=\text { t'es, } L \\
=\text { elle }\end{array}$ & $\begin{array}{l}\text { Tи } \Delta ?=\text { Ty } D ?=\text { Th } \Delta e ?=T y \\
\text { de? }=\text { Tu es où ? }\end{array}$ \\
\hline rébus & 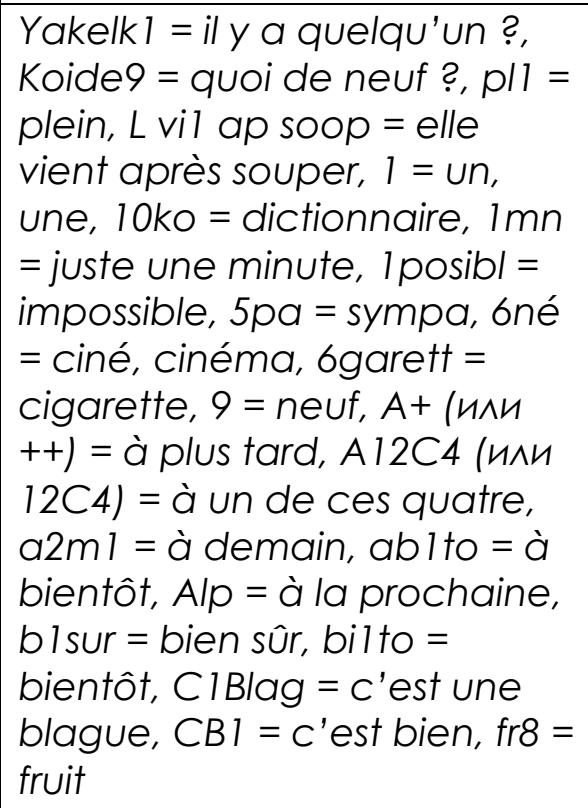 & 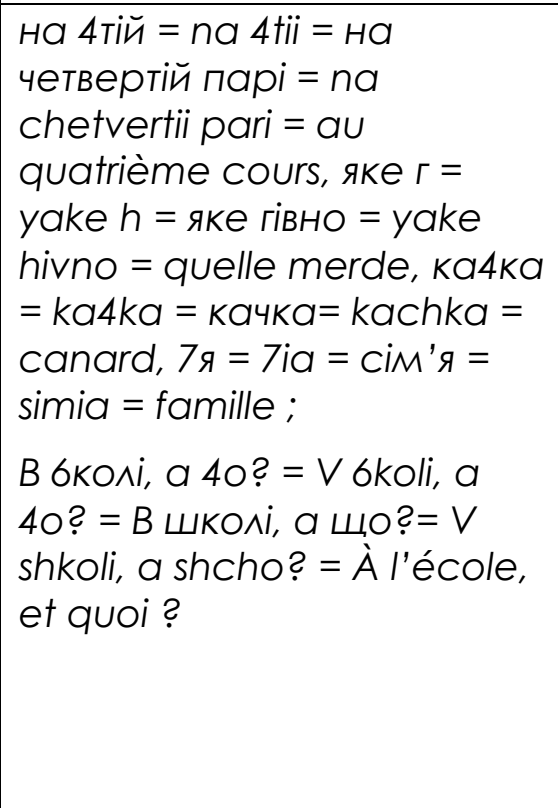 \\
\hline $\begin{array}{l}\text { suppression de lettres } \\
\text { jugées facultatives }\end{array}$ & $\begin{array}{l}\text { slt = salut, } d s l=\text { désolé, } b c p= \\
\text { beaucoup, bb = bébé, tjrs = } \\
\text { toujours, bjr = bonjour, } \mathrm{mm}= \\
\text { même, cmb combien, } \mathrm{pr}= \\
\text { pour, pkoi = pourquoi, bsr = } \\
\text { bonsoir, Cc = coucou, fR = } \\
\text { faire, CT = c'était, dsl = } \\
\text { desolé, frR = frère, GT = j'étais, } \\
\text { HT = acheter, ID = idée, IR = } \\
\text { hier, JC = je sais, JMS = jamais, } \\
\text { jr = jour, jtdr = je t'adore, JTM } \\
=\text { je t'aime, jv = je vais, Igtmps }\end{array}$ & $\begin{array}{l}\text { пн = pn = понеАілок = } \\
\text { ponedilok = lundi, xз = hz = } \\
\text { хаза = квартира = } \\
\text { арpartement, пт = pt = } \\
\text { п'ятниця = piatnytsia = } \\
\text { vendredi, спс = sps = } \\
\text { списати = spysaty = copier, } \\
\text { ск = sk = скільки = skilky = } \\
\text { combien, крч = krch = } \\
\text { короче = koroche = bref, } 43 \\
=\text { chz = через = cherez = à } \\
\text { travers }\end{array}$ \\
\hline soudure & $\begin{array}{l}\text { TaKpaC = tu n'as qu'à } \\
\text { passer; menfin = m'enfin = }\end{array}$ & - \\
\hline
\end{tabular}




\begin{tabular}{|c|c|c|}
\hline & mais enfin & \\
\hline anglicismes & $\begin{array}{l}\text { CUL8R = see you later, LOL Ipl, } \\
\text { asap = aussi vite que possible }\end{array}$ & 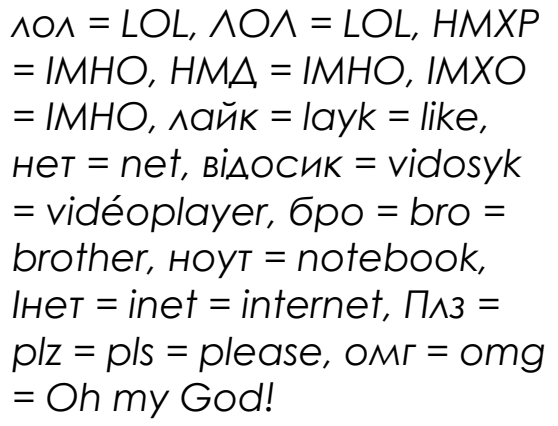 \\
\hline $\begin{array}{l}\text { francisation / } \\
\text { Ukrainisation des } \\
\text { anglicismes }\end{array}$ & $\begin{array}{l}\text { LOL devient MDR (mort de } \\
\text { rire), ravie q on é pu se see, } \\
\text { j'y go = je dois partir }\end{array}$ & $\begin{array}{l}\text { твітнути = twitnouté = twitter, } \\
\text { ^айкати = laykaté = liker, } \\
\text { гуглити = huhlyty = googler, } \\
\text { сорян = sorian = sorry, } \\
\text { мемчик = memchyk = } \\
\text { meme, opoткати = fotkaty = } \\
\text { faire de photos, opoтик = } \\
\text { fotyk = appareil photo }\end{array}$ \\
\hline $\begin{array}{l}\text { extension graphique } \\
\text { expressive }\end{array}$ & $\begin{array}{l}\text { Jtadooooooooooore, } \\
\text { Gtèèèèèèèèèmmmmmmmm }\end{array}$ & 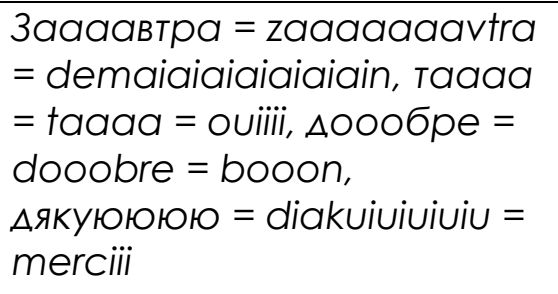 \\
\hline troncations & $\begin{array}{l}\text { ordi = ordinateur, lut = salut, } \\
\text { tain = putain, auj = } \\
\text { aujourd'hui, BIZ = bisous, bises, } \\
\text { DAC = d'accord }\end{array}$ & 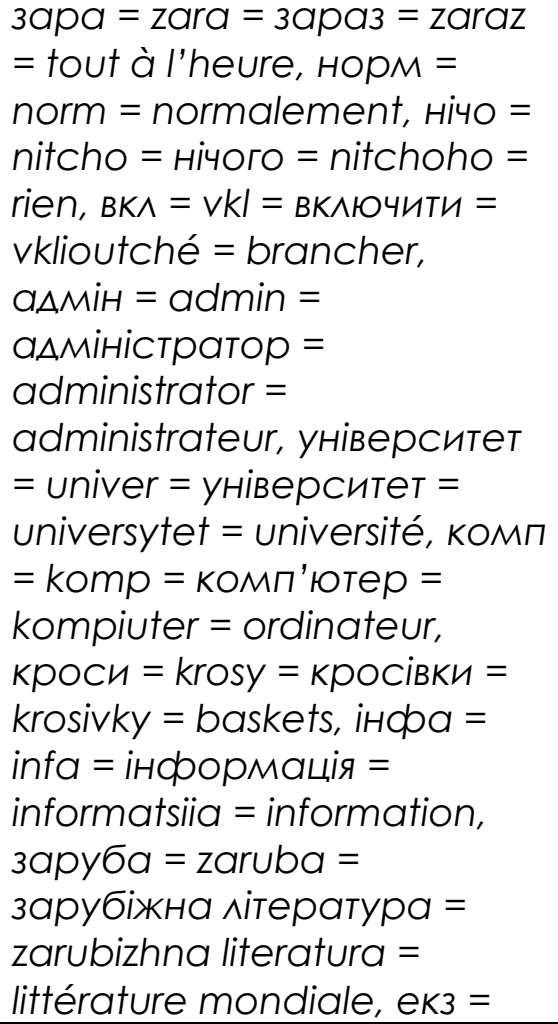 \\
\hline
\end{tabular}




\begin{tabular}{|c|c|c|}
\hline & & 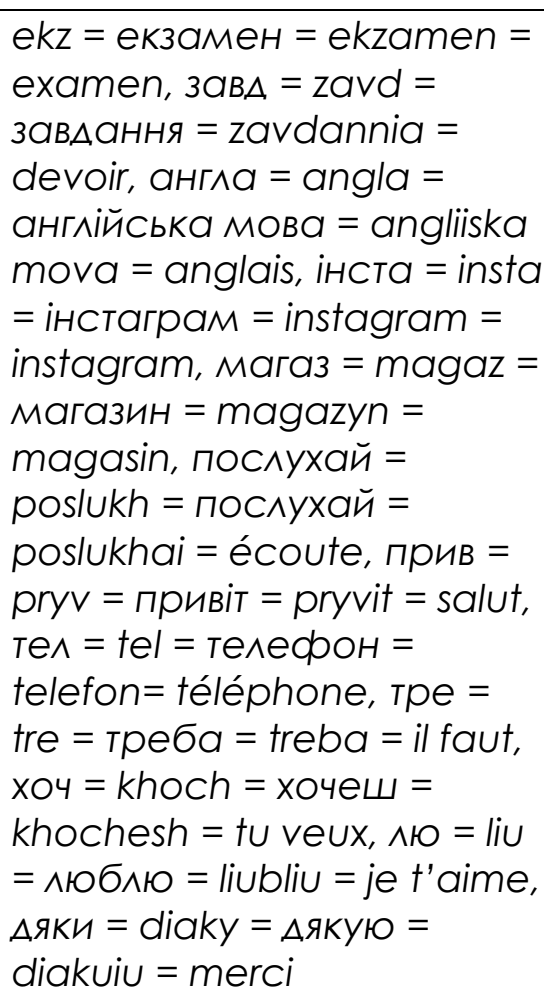 \\
\hline verlan & $\begin{array}{l}\text { ya pa de meufs = il n'y a pas } \\
\text { de femmes }\end{array}$ & - \\
\hline $\begin{array}{l}\text { squelettes } \\
\text { consonantiques }\end{array}$ & $\begin{array}{l}t+=\text { tout, } d s=\text { dans, lgtps }= \\
\text { longtemps, pb, prb = } \\
\text { problème, vs = vous }\end{array}$ & - \\
\hline $\begin{array}{l}\text { syllabogrammes et } \\
\text { rébus }\end{array}$ & $\begin{array}{l}\text { I = elle, c = c'est, sais/sait, } \\
\text { s'est, c'é[tait], } d=\text { des, } g= \\
\text { j'ai, } v=\text { vais }\end{array}$ & - \\
\hline logogrammes & $\begin{array}{l}l=\text { in, un, } 2=\text { de, } 2 m 1= \\
\text { demain, a2line = Adeline, } \\
\text { abilto = à bientôt }\end{array}$ & - \\
\hline agglutination & $Y a=y a$ & - \\
\hline $\begin{array}{l}\text { suppression de } \\
\text { l'apostrophe }\end{array}$ & dabor $=$ d'abord & - \\
\hline $\begin{array}{l}\text { variantes vocaliques } \\
\text { ou semi-vocaliques }\end{array}$ & $\begin{array}{l}\text { moa }=\text { moi, toa }=\text { toi, ui }=\text { oui, } \\
\text { mouais }=\text { oui }\end{array}$ & - \\
\hline
\end{tabular}

\section{Conclusion}

La comparaison des e-langues des jeunes fait ressortir des traits communs seulement dans les aspects suivants: suppression de lettres jugées facultatives, siglaison, soudure, anglicismes calqués, francisation / ukrainisation des anglicismes, extension graphique expressive et troncation. 
Les résultats de notre étude de plus de 250 éléments des e-langues des jeunes ont mis en évidence le fait qu'Internet offre un immense espace public à la production langagière familière, à des pratiques du français et de l'ukrainien affranchies de toute règle, à l'écrit libre et libéré. L'écrit en français et en ukrainien devient familier, s'oralise. Cela enrichit l'espace d'expression de la langue ordinaire, la capacité expressive de l'écrit. Grâce à Internet, on peut faire avec l'écrit des choses qu'on ne pouvait pas faire avant.

Nous croyons que la e-langue des jeunes, ce davantage en France qu'en Ukraine, a une influence sur la langue ordinaire, mais qu'elle ne la modifie pas directement, parce que la langue ordinaire n'existe pas comme unité homogène. On ne peut imaginer qu'un tel média soit linguistiquement neutre. Bien sûr, il y a des mots nouveaux propres à la pratique d'Internet, principalement des anglicismes.

Enfin, nous voudrions souligner le fait que les gens qui affirment que le e-langage appauvrit la langue parce qu'il n'est rien d'autre qu'un charabia inventé et sans règles se trompent. La communication par Internet provoque l'évolution de la langue commune des jeunes, en France et en Ukraine : il ne faut pas en avoir peur, mais utiliser ces procédés de formation de mots et d'expressions, d'unités langagières afin d'enrichir la langue ordinaire.

\section{Bibliographie}

ANIS, J. (1999) : Internet, communication et langue française. Paris: Hermès Sciences publications.

- (2003) : Communication électronique scripturale et formes langagières. Actes des Quatrièmes Rencontres Réseaux Humains. Poitiers : Université de Poitiers, pp. 57-70. Disponible en ligne : http://edel.univ-poitiers.fr/rhrt/document547.php [consulté le 10.06.2017].

BALDINO PUTZKA, M. \& E. BOUTIN (2012) : La Cyberlangue dans les forums de discussion : étude exploratoire dans le domaine de la télé réalité. Colloque de la SFSIC. Rennes: Université de Rennes, pp.1-7.

BARTHELEMY, F. (2007) : Cunéiforme et SMS : analyse graphémique de systèmes d'écriture hétérogènes. Actes du 26ème Colloque international sur le Lexique et la Grammaire. Marne-la-Vallée : Universite Paris-Est Marne-la-Vallee, pp. 1-8. Disponible en ligne : http://infolingu.univ-mlv.fr/Colloques/Bonifacio/actes.html [consulté le 12.05.2017].

BEREGOVSKAYA, E. (1996) : Youth Slang: the formation and functioning. Questions on linguistics, 3, pp. 32-41.

BOYER, H. (2001) : Le français des jeunes vécu/vu par les étudiants. Enquêtes à Monpellier, Paris, Lille. Langage \& société, 95, pp. 75-87.

CHEMERKIN, C. (2009) : Ukrainian language on the Internet: the extra-linguistic and internal structural processes. Kyiv : PPVF.

DEJOND, A. (2006) : Cyberlangage. Bruxelles : Éditions Racine. 
FIÉVET, A.-C., A. PODHORNÁ-POLICKÁ \& S. STAROBOVÁ (2010) : Circulation des néologismes argotiques chez les jeunes locuteurs : facteurs déchanges entre les idiolectes et les sociolectes au niveau endo-groupal et exo-groupal. Actes del I Congrés Internacional de Neologia de les Llengües Romàniques. Barcelona: IULA, Universitat Pompeu Fabra, pp. 983-998.

GADET, F. (2003) : La variation sociale. Paris : Ophrys.

GAJOS, M. (2006) : Parler en écrivant: le lexique des internautes français. Verbum, Vol. 8, Issue 1, pp. 171-180.

GOUDET, L. (2016) : Spécifités des nouveaux discours en ligne. Disponible en ligne : http://eriac.univ-rouen.fr/specificites-des-nouveaux-discours-en-ligne/

KONDRATYUK, O. (2005) : Youth slang as a linguistic phenomenon. Independent cultural and scientific journal "ï", 38, pp. 212-220.

OSTAPENKO, G. (2013) : The youth virtual communication features on the Internet. The world of social communication, 10, pp. 113-117.

PODHORNÁ-POLICKÁ, A. (2007) : Peut-on parler d'un argot des jeunes ? : analyse lexicale des universaux argotiques du parler de jeunes en lycées professionnels en France (Paris, Yzeure) et en République tchèque (Brno). Thèse en cotutelle. Paris Brno : Université René Descartes, Université Masaryk.

- (2008) : Dynamique socioculturelle et médias : causes et conséquences de la médiatisation de la «langue des jeunes ». Echo des études romanes, Vol. IV, Num. 2, pp. 23-38.

SOURDOT, M. (2003) : La dynamique du langage des jeunes. Des mots des jeunes au langage scolaire. Résonances, 10, pp.4-5.

YAROSHCHUK, L. (2012) : Computer slang as a form of youth communication. Actual problems of Romano-Germanic philology and applied linguistics, 1, pp. 156-164.

\section{Sitographie}

https://autodidacteblog.wordpress.com/2016/07/26/langage-sms/

http://www.jeuxvideo.com/forums/1-50-133420277-1-0-1-0-sa-veut-dire-quoicmb.htm 